\title{
Relación entre el promedio escolar y las diferentes bandas de frecuencia del electroencefalograma de estudiantes de Medicina de la UJED campus Durango
}

\section{Relationship between the school average and the different frequency bands of the electroencephalogram of medical students of the UJED Campus Durango}

\author{
RIOS-VALLES, José Alejandro†*',", BARRAGAN-LEDESMA, Laura Ernestina", ESTRADA- \\ MARTÍNEZ, Sergio y POBLANO, Adrián' \\ Instituto de Investigación Científica de la Universidad Juárez del Estado de Durango. Calle Constitución 404, Zona \\ Centro, 34100 Durango, Dgo \\ "Facultad de Medicina y Nutrición de la Universidad Juárez del Estado de Durango. Avenida Universidad s/n, Los \\ Ángeles, 34076 Durango, Dgo \\ "'Instituto Nacional de Rehabilitación, SS. Calz México-Xochimilco 289, Coapa, Arenal Tepepan, Tlalpan, 14389 Ciudad \\ de México, CDMX
}

ID $1^{\text {er }}$ Autor: José Alejandro, Ríos-Valles / ORC ID: 0000-0002-8407-3017, Researcher ID Thomson: X-3209-2018, CVU CONACYT ID: 313266

ID $1^{\text {er }}$ Coautor: Laura Ernestina, Barragán-Ledesma / ORC ID: 0000-0001-5929-1648, Researcher ID Thomson: X9239-2018, CVU CONACYT: 204908

ID $2^{\text {do }}$ Coautor: Sergio, Estrada-Martínez / ORC ID: 0000-0002-7633-6841, Researcher ID Thomson: T-1487-2018, CVU CONACYT ID: 393364

ID $3^{\text {er }}$ Coautor: Adrián, Poblano / ORC ID: 0000-0002-1178-8900, PubMed Author ID: poblano a, CVU CONACYT ID: 14343

DOI: $10.35429 /$ JOTE.2019.10.3.32.39

Recibido 02 de Noviembre, 2019; Aceptado 28 de Diciembre, 2019

\begin{abstract}
Resumen
Objetivos: Identificar la relación de las diferentes bandas de frecuencia de la actividad cerebral registrada en cada electrodo del electroencefalograma cuantitativo (QEEG) y el promedio escolar en estudiantes de la carrera de medicina de la Facultad de Medicina y Nutrición de la Universidad Juárez del Estado de Durango. Metodología: Investigación analítica, transversal, retrolectiva, descriptiva y correlacional entre el promedio escolar y las diferentes bandas de frecuencia del electroencefalograma obtenido de 285 expedientes de estudiantes de Medicina de la UJED Campus Durango seleccionados en un muestreo no probabilístico y por conveniencia previa firma de consentimiento informado. Contribución: El Alpha de Chronbach de los datos analizados fue de .958. Se identificó correlación significativa entre el promedio escolar y la banda theta de la actividad cerebral en los electrodos FP1(r=-.13 p=.02), FP2 (r=-.16 p=.004), O1 $(\mathrm{r}=-.12 \mathrm{p}=.03), \mathrm{F} 7(\mathrm{r}=-.14 \mathrm{p}=.01), \mathrm{T} 3(\mathrm{r}=-.11 \mathrm{p}=.04)$ y T4 $(\mathrm{r}=-11 \mathrm{p}=.04)$ definiéndose que a menor actividad Theta existe mejor promedio escolar ó que a mayor actividad Theta se tiene menor promedio escolar, lo que se ve como una oportunidad de intervención. En los demás electrodos de registro de la actividad Theta y las demás bandas de frecuencia de la actividad cerebral no presentaron correlación significativa.
\end{abstract}

Electroencefalograma cuantitativo, Promedio escolar, Estudiantes de medicina

\begin{abstract}
Objectives: To identify the relationship of the different frequency bands of brain activity recorded in each electrode of the quantitative electroencephalogram (QEEG) and the school average in students of the medical career of the Faculty of Medicine and Nutrition of the Juarez University of the State of Durango. Methodology: Analytical, crosscutting, retrolective, descriptive and correlational research between the school average and the different frequency bands of the electroencephalogram obtained from 285 medical student records of the UJED Campus Durango selected in a non-probabilistic sampling and at the convenience of signature of informed consent. Contribution: The Chronbach Alpha from the analyzed data was .958. Significant correlation was identified between the school average and the theta band of brain activity in the electrodes FP1(r=-.13 p=.02), FP2 $(r=-.16$ $\mathrm{p}=.004), \mathrm{O} 1(\mathrm{r}=-.12 \mathrm{p}=.03), \mathrm{F} 7(\mathrm{r}=-.14 \mathrm{p}=.01)$, T3 (r=-.11 p=-.04) and $\mathrm{T} 4(\mathrm{r}=-.11 \mathrm{p}=-.04)$ defining that at lower Theta activity exists better school average or that the higher the has the lower school average, which is seen as an opportunity for intervention. In the other electrodes recording Theta activity and the other frequency bands of brain activity they did not have significant correlation.
\end{abstract}

Quantitative Electroencephalogram, School Average, Medical Students

Citación: RIOS-VALLES, José Alejandro, BARRAGAN-LEDESMA, Laura Ernestina, ESTRADA-MARTÍNEZ, Sergio y POBLANO, Adrián. Relación entre el promedio escolar y las diferentes bandas de frecuencia del electroencefalograma de estudiantes de Medicina de la UJED campus Durango. Revista de Educación Técnica. 2019. 3-10: 32-39.

\footnotetext{
*Correspondencia al Autor (Correo Electrónico: alexriva@hotmail.com)
}

$\dagger$ Investigador contribuyendo como primer autor. 


\section{Introducción}

En todo estudiante universitario la integridad funcional de su encéfalo es esencial para el adecuado desempeño de las funciones mentales relacionadas con el aprendizaje, las cuales son indispensables para un desarrollo escolar con satisfactoria eficiencia terminal. Esta última es un importante indicador que determina la eficiencia de los procesos educativos en las universidades, y que la Secretaría de Educación Pública la define como el número de alumnos que terminan un nivel educativo de manera regular (dentro del tiempo ideal establecido) y el porcentaje de alumnos que lo culminan extemporáneamente. (Secretaria de Educacion Publica, 2014). En el ciclo escolar 2014-2015 el gasto promedio por alumno fue de $72.7 \mathrm{mil}$ pesos para la educación superior. (Gobierno Federal de los Estados Unidos Mexicanos, 2015) La Secretaria de Educación Pública del Gobierno de los Estados Unidos Mexicanos, en su IV Informe de Labores del ciclo escolar 2016-2017 informa que el gasto promedio general por alumno en Educación Superior fue de 77.5 mil pesos y en el ciclo 2017-2018 fue de 79.9 mil pesos por alumno (Secretaria de Educación Pública, México Gobierno de la República, 2016) (Gobierno de los Estado Unidos Mexicanos, 2017) (Gobierno de los Estados Unidos Mexicanos, 2018)

A partir del año de 2013 al 2018, la eficiencia terminal de alumnos de la Facultad de Medicina y Nutrición ha mostrado poco cambio pues de iniciar con el 46\% en el 2013 culmina con el $50 \%$ en el ciclo Ago-Dic del 2018, como se puede observar en la tabla 1. (Universidad Juárez del Estado de Durango, 2015) (Universidad Juárez del Estado de Durango, 2016) (Universidad Juárez del Estado de Durango, 2017) (Universidad Juárez del EsTado de Durango, 2018).

\begin{tabular}{|c|c|c|c|}
\hline Ciclo & Ingreso & Terminaron & $\begin{array}{c}\text { Eficiencia } \\
\text { terminal }\end{array}$ \\
\hline 2013 & 159 & 73 & $46 \%$ \\
\hline 2014 & 170 & 66 & $39 \%$ \\
\hline 2015 B (Ene-Jul) & 101 & 40 & $40 \%$ \\
\hline 2015 A (Ago-Dic) & 113 & 40 & $35 \%$ \\
\hline 2016 B (Ene-Jul) & 120 & 46 & $38 \%$ \\
\hline 2016 A (Ago-Dic) & 139 & 48 & $35 \%$ \\
\hline 2017 B (Ene-Jul) & 143 & 68 & $47 \%$ \\
\hline 2017 A (Ago-Dic) & 132 & 63 & $48 \%$ \\
\hline 2018 B (Ene-Jul) & 143 & 68 & $48 \%$ \\
\hline 2018 A (Ago-Dic) & 130 & 65 & $50 \%$ \\
\hline
\end{tabular}

Tabla 1 Eficiencia terminal 2013-2018 FAMEN

Fuente: Anuarios estadísticos UJED 2015, 2016, 2017, 2018

ISSN-2523-2460

ECORFAN $^{\circledR}$ Todos los derechos reservados

\section{Electroencefalografía}

En la actualidad son pocos los estudios que relacionen información de la actividad cerebral mediante electroencefalografía cuantitativa y el desempeño escolar en personas sin un presunto problema neurológico previo (Mulas, Hernandez, Mattos, \& Abad - Mas, 2006) (Salvatierra Belén, 2002) (Janzen, Graap, Stephanson, Marshall, \& Fitzsimmons, 1995).

Un estudio cuasi experimental en la Universidad de Tennessee reporta que estudiantes de su comunidad participaron para incrementar su actividad electroencefalográfica (Beta, Alfa y Tetha) y la eficiencia de su atención mediante una técnica de Biofeedback, teniendo pobres resultados (Lubar, Rasey, McIntyre, Zoffuto, \& Abbott, 2014). Así mismo, en la ciudad de Durango, México, se evaluaron alumnos del primer semestre del área de las ciencias de la salud de la UJED, confirmándose actividad cerebral lenta, del rango delta, en el $29 \%$ de los participantes con menor desempeño escolar (Rios Valles, Barragan Ledesma, \& Flores Saucedo, UJED, 2013).

La electroencefalografía cuantitativa (qEEG) es una técnica neurofisiológica no invasiva, que procesa la señal eléctrica del electroencefalograma (EEG) convencional y cuantifica la distribución relativa de cada frecuencia, con la ventaja de su gran resolución temporal y la gran posibilidad de estudiar cómo definir desviaciones de la normalidad en el funcionamiento cerebral de un individuo (Damas Lopez, Martín Rodríguez, \& León Carrión, 2005).

El EEG permite conocer las características de la actividad electrofisiológica cerebral (Ysunza \& Perusquia Ortega, 2007) (Díaz Martínez C, 2006) (Casas Fernández C, 2002) y ésta señal del EEG puede ser medida a través del cuero cabelludo (Niedermeyer, Lopes da Silva, \& Schomer, 2005).

Las fluctuaciones rítmicas en la señal EEG pueden ocurrir dentro de diferentes bandas de frecuencia (Marosii \& al., 2002) (Lutsyuk, Éismont, \& Pavlenko, 2006) (Berka, 2007) (Baker \& al., 2010), que a continuación se describen:

RIOS-VALLES, José Alejandro, BARRAGAN-LEDESMA, Laura Ernestina, ESTRADA-MARTÍNEZ, Sergio y POBLANO, Adrián. Relación entre el promedio escolar y las diferentes bandas de frecuencia del electroencefalograma de estudiantes de Medicina de la UJED campus Durango. Revista de Educación Técnica. 2019 
Las ondas Alfa poseen frecuencias entre 8 y $13 \mathrm{~Hz}$. Se registran en sujetos normales despiertos, en estado de reposo o sin actividad y con los ojos cerrados, localizándose en la zona occipital; su amplitud va de 20 a $200 \mu \mathrm{V}$.

Las ondas Beta poseen frecuencias entre 14 y $30 \mathrm{~Hz}$, pueden llegar hasta los $50 \mathrm{~Hz}$; se registran en las regiones parietal y frontal.

Las ondas Theta poseen frecuencias entre 4 y $7 \mathrm{~Hz}$ y se presentan en la infancia aunque también pueden presentarlas los adultos en períodos de stress emocional y frustración. Se localizan en las zonas parietal y temporal.

Las ondas Delta poseen frecuencias inferiores a $3.5 \mathrm{~Hz}$ y se presentan durante el sueño profundo, en la infancia $y$ en enfermedades orgánicas cerebrales graves. (Barea Navarro, 2014)

\section{Desempeño Escolar}

En el proceso del aprendizaje se debe considerar al sistema nervioso central (SNC) como el responsable de las funciones necesarias para el desempeño escolar, ya que este enfrenta una constante adaptación al ambiente mediante procesos de neuroplasticidad (Portellano, 2005) y es un sistema biológico complejo relacionado con los procesos y funciones propios del aprendizaje. (Flores Lázaro \& Ostrosky Solis, 2008) (Fuster, 2002) (Muñoz Céspedes \& Tirapu Ustárroz, 2001)

El desempeño escolar se ha definido como el "cumplimiento de las metas, logros u objetivos establecidos en el programa o asignatura (Velez-Van, 2005), o como el nivel de logro que puede alcanzar un estudiante en una o varias asignaturas (Barceló, 2006), la variable que es más usada para medir el desempeño escolar son las calificaciones escolares. (Edel, 2003) (Rodriguez Ayán, 2011)

El desempeño escolar es una variable compleja que depende de circunstancias internas y externas, lo que permite que pueda ser predicho por características internas como las cognoscitivas (inteligencia) y del comportamiento (personalidad, motivación). (Cupani, 2013)
Como evidencia del aprendizaje, el desempeño escolar puede ser expresado por la calificación asignada por el profesor o el promedio obtenido por el alumno. El promedio resume el rendimiento escolar y los problemas escolares relacionados con el bajo rendimiento y la deserción académica son de interés para padres, maestros y profesionales en el campo educativo y pueden estar relacionados con aspectos como la salud física y mental. (Palacios, 2007)

El aprendizaje depende de la adecuada función del sistema nervioso y el proceso de aprender involucra la organización de redes neurales por lo que se debe considerar que los trastornos del aprendizaje se manifiestan por dificultades en el desempeño escolar.

La medición de la inteligencia y las habilidades cognitivas ha sido estudiada por diferentes autores desde principios del siglo pasado. La evaluación de este constructo consiste en muestrear una amplia variedad de habilidades y combinar los puntajes obtenidos en un puntaje compuesto adecuado, que generalmente es referido como coeficiente de inteligencia. (Echavarri, 2007).

Por todo lo anteriormente descrito y particularmente observando que la eficiencia terminal de los alumnos de la Facultad de Medicina y Nutrición de la Universidad Juárez del Estado de Durango, es menor a la media nacional, referida en el Plan Nacional de Desarrollo del 2007, resulta de importancia el tratar de identificar si la actividad cerebral tiene alguna diferencia entre alumnos con desempeño escolar alto, moderado y bajo.

\section{Planteamiento del problema}

La baja eficiencia terminal en estudiantes de Medicina es un problema que preocupa a cualquier Universidad o Institución Educativa en cualquier parte del mundo, pues el rezago escolar evidenciado por la baja eficiencia terminal representa un costo económico y social importante para cualquier país.

De acuerdo a la estimación referida en el VI Informe de Gobierno del 2018, cada alumno en educación superior representa un gasto de 79.9 mil pesos anuales, lo cual representa un gasto importante para el país. 


\section{Justificación}

Por lo expresado en el planteamiento del problema, es que se requiere la realización de investigaciones que ayuden a identificar si el funcionamiento de él órgano responsable de las funciones neurocognitivas, el cerebro, requiere de alguna estrategia de atención específicamente orientada para optimizar su desempeño, conociendo las características de la actividad cerebral en los estudiantes con diferentes niveles de desempeño escolar.

Al identificar las características de la actividad electrofisiológica cerebral en estudiantes de medicina, se espera reconocer en la electroencefalografía cuantitativa (QEEG) un instrumento confiable para apoyar la detección de alumnos que requieran atención específica para apoyar sus procesos de aprendizaje durante su formación profesional como médicos, lo cual a su vez permitiría un mejor uso de los recursos económicos destinados a la educación superior.

\section{Pregunta de investigación}

¿Cuál es la relación entre la actividad cerebral y el desempeño escolar en estudiantes de medicina de la Facultad de Medicina y Nutrición de la Universidad Juárez del Estado de Durango?

\section{Hipótesis alterna}

La actividad cerebral se relaciona con el desempeño escolar en estudiantes de medicina de la Facultad de Medicina y Nutrición de la Universidad Juárez del Estado de Durango.

\section{Objetivo general}

Identificar la correlación de las diferentes bandas de frecuencia de la actividad cerebral y su amplitud respectiva registrada en cada electrodo del electroencefalograma cuantitativo (QEEG) y el desempeño escolar en alumnos de la carrera de medicina de la FAMEN.

\section{Objetivos específicos}

1. Conocer la media de cada una de las bandas de frecuencia de la actividad cerebral registrada en cada electrodo del electroencefalograma cuantitativo (QEEG) en el grupo de alumnos con promedio escolar alto.
2. Identificar la media de cada una de las bandas de frecuencia de la actividad cerebral registrada en cada electrodo del electroencefalograma cuantitativo (QEEG) en el grupo de alumnos con promedio escolar moderado.

3. Establecer la media de cada una de las bandas de frecuencia de la actividad cerebral registrada en cada electrodo del electroencefalograma cuantitativo (QEEG) en el grupo de alumnos con promedio escolar bajo.

\section{Metodología}

La investigación realizada fue de tipo exploratoria, no experimental, transversal, retrospectiva, retrolectiva, descriptiva y correlacional de un muestreo no probabilístico, obtenido por conveniencia, con firma previa de consentimiento informado, con un $95 \%$ de confiabilidad en participantes voluntarios de un universo de 1139 estudiantes de la carrera de Medicina, de la FAMEN, DGO. UJED.

\section{Criterios de inclusión}

1. Todos los alumnos de la carrera de Medicina, de todos los semestres o ciclos escolares, que acepten participar voluntariamente previa firma de consentimiento informado.

\section{Criterios de exclusión}

1. Alumnos que tengan antecedentes de algún padecimiento neurológico o psiquiátrico, aunque en respeto a los derechos humanos se les realizarán los estudios correspondientes, pero no serán incluidos en la muestra de estudio.

\section{Criterios de eliminación}

1. Alumnos cuya información clínica o del desempeño escolar esté incompleta. 


\section{Procedimiento}

A 285 estudios de QEEG realizados a estudiantes de la carrera de Medicina, durante el periodo comprendido del mes de agosto del 2013 al mes de Diciembre de 2015, que aceptaron, previo consentimiento informado, participar de manera voluntaria y por conveniencia en la conformación de la muestra de estudio, se les realizó el análisis de frecuencias y amplitudes mediante el Software del equipo Neuron Spectrum 4P para identificar la media de las diferentes bandas de frecuencia y sus respectivas amplitudes obtenidas en cada uno de los 16 electrodos empleados para el registro de la actividad cerebral de cada participante.

Con esta información se conformó la base de datos para su análisis descriptivo y correlacional mediante el software Statistical Package for de Social Sciences (SPSS) versión 20.0.

\section{Aspectos éticos}

De acuerdo al artículo 17 de la la Ley General de Salud en México la investigación realizada fue un estudio sin riesgo para la salud y en la que se consideró el artículo 20 de la misma Ley, respetando el consentimiento informado de todos los participantes y la Declaración de Helsinki.

\section{Resultados}

El total de los estudios electroencefalográficos valuados fue de 285, 122 correspondieron a personas del sexo masculino $(42.8 \%)$ y 163 al sexo femenino $(57.2 \%)$.

La media de la edad en años del grupo estudiado fue de 22.37, Desviación estándar de 2.28, valor mínimo de 18 y máximo de 43 . El promedio escolar del grupo en general mostró; Media 8.56; Desviación Estándar 0.4; Valor mínimo 7.5 y Valor máximo 9.70. La confiabilidad de los datos del promedio escolar referido por los encuestados y los valores de la amplitud y la frecuencia en los 285 estudios electroencefalográficos mediante el Alpha de Cronbach fue de 0.95 (95\%).

Agrupando a los participantes por su Estatus Escolar, 143 correspondieron a no regulares $(50.2 \%)$ y $142(49.8 \%)$ a Regulares.
La distribución de los participantes por promedio escolar se agrupó considerando a los de promedio menor a quienes refirieron un promedio menor a la media menos una desviación estándar, los de promedio moderado a quienes refirieron un promedio en la media mas menos una desviación estándar y los de promedio alto quienes refirieron promedio por arriba de la media mas una desviación estándar. (ver tabla 2)

\begin{tabular}{|l|r|r|}
\hline \multicolumn{1}{|c|}{ Promedio escolar } & \multicolumn{1}{c|}{ N } & \multicolumn{1}{c|}{} \\
\hline Menor $(<8.10)$ & 41 & 14 \\
\hline Moderado (entre 8.96 y 8.10) & 194 & 69 \\
\hline Alto $(>8.96)$ & 50 & 17 \\
\hline Total & 285 & 100 \\
\hline
\end{tabular}

Tabla 2 Actividad Cerebral y Desempeño Escolar por promedio

El número y proporción de alumnos por semestre se observa en la tabla 3.

\begin{tabular}{rr|r|r|}
\multicolumn{1}{|c|}{ Grado } & \multicolumn{2}{c|}{ Precuencia } & \multicolumn{1}{c|}{ Porcentaie } \\
\hline 2 & 1 & 0.4 \\
\hline 3 & 28 & 9.1 \\
\hline 4 & 15 & 5.3 \\
\hline 5 & 68 & 23.9 \\
\hline 6 & 27 & 9.5 \\
\hline 7 & 25 & 8.8 \\
\hline 8 & 10 & 3.5 \\
\hline 9 & 50 & 17.5 \\
\hline 10 & 63 & 22.1 \\
\hline Total & 285 & 100 \\
\hline
\end{tabular}

Tabla 3 Grado Escolar

Mediante Correlación de Spearman se observó que de las diferentes bandas de frecuencia de la actividad electroencefalográfica solo la banda Theta de las áreas FP1, FP2, F7, $\mathrm{T} 3, \quad \mathrm{~T} 4$ y $\mathrm{O} 1$ muestra correlación estadísticamente significativa (ver tabla 4).

\begin{tabular}{|l|l|r|}
\hline \multirow{2}{*}{ FP1HzM } & $\mathrm{r}$ & \multicolumn{2}{c|}{ Promedio } \\
\cline { 2 - 3 } & $\mathrm{p}$ & -.13 \\
\hline \multirow{2}{*}{ FP2HzM } & $\mathrm{r}$ & .02 \\
\cline { 2 - 3 } & $\mathrm{p}$ & -.16 \\
\hline \multirow{2}{*}{ O1HzM } & $\mathrm{r}$ & .004 \\
\cline { 2 - 3 } & $\mathrm{p}$ & -.12 \\
\hline \multirow{2}{*}{ F7HzM } & $\mathrm{r}$ & .03 \\
\cline { 2 - 3 } & $\mathrm{p}$ & -.14 \\
\hline \multirow{2}{*}{ T3HzM } & $\mathrm{r}$ & .01 \\
\cline { 2 - 3 } & $\mathrm{p}$ & -.11 \\
\hline T4HzM & $\mathrm{r}$ & .04 \\
\cline { 2 - 3 } & $\mathrm{p}$ & -.118 \\
\hline
\end{tabular}

Tabla 4 Correlación Spearman

Las demás bandas de frecuencia de la actividad electroencefalográfica no mostraron correlación estadísticamente significativa.

RIOS-VALLES, José Alejandro, BARRAGAN-LEDESMA, Laura Ernestina, ESTRADA-MARTÍNEZ, Sergio y POBLANO, Adrián. Relación entre el promedio escolar y las diferentes bandas de frecuencia del electroencefalograma de estudiantes de Medicina de la UJED campus Durango. Revista de Educación Técnica. 2019 
La media de las diferentes bandas de frecuencia en cada grupo, según el promedio escolar, mostraron valores similares entre sí. (Ver tablas 5, 6 y 7)

\begin{tabular}{|l|r|r|r|r|r|}
\hline \multicolumn{1}{r|}{ DELT } & \multicolumn{1}{c}{ THET } & \multicolumn{1}{c|}{ ALF } & \multicolumn{1}{c|}{ BETA } & \multicolumn{1}{c|}{ BETA } \\
\hline FP1Hz & 1.17 & 5.02 & 10.1 & 16.14 & 24.68 \\
\hline $\mathrm{FP} 2 \mathrm{~Hz}$ & 1.14 & 5.19 & 10.1 & 16.21 & 24.85 \\
\hline $\mathrm{F} 3 \mathrm{~Hz}$ & 1.30 & 5.44 & 10.1 & 16.20 & 24.22 \\
\hline $\mathrm{F} 4 \mathrm{~Hz}$ & 1.27 & 5.45 & 10.1 & 16.23 & 24.38 \\
\hline $\mathrm{C} 3 \mathrm{~Hz}$ & 1.31 & 5.44 & 10.1 & 16.26 & 24.27 \\
\hline $\mathrm{C} 4 \mathrm{~Hz}$ & 1.30 & 5.41 & 10.1 & 16.25 & 24.28 \\
\hline $\mathrm{P} 3 \mathrm{~Hz}$ & 1.31 & 5.41 & 10.3 & 16.12 & 23.91 \\
\hline $\mathrm{P} 4 \mathrm{~Hz}$ & 1.28 & 5.40 & 10.3 & 16.09 & 24.08 \\
\hline $\mathrm{O} 1 \mathrm{~Hz}$ & 1.31 & 5.43 & 10.6 & 15.93 & 23.85 \\
\hline $\mathrm{O} 2 \mathrm{~Hz}$ & 1.30 & 5.26 & 10.7 & 15.85 & 23.88 \\
\hline $\mathrm{F} 7 \mathrm{~Hz}$ & 1.24 & 5.30 & 10.1 & 16.35 & 24.62 \\
\hline $\mathrm{F} 8 \mathrm{~Hz}$ & 1.22 & 5.35 & 10.2 & 16.35 & 24.92 \\
\hline $\mathrm{T} 3 \mathrm{~Hz}$ & 1.25 & 5.36 & 10.2 & 16.46 & 24.89 \\
\hline $\mathrm{T} 4 \mathrm{~Hz}$ & 1.28 & 5.38 & 10.2 & 16.52 & 24.82 \\
\hline $\mathrm{T} 5 \mathrm{~Hz}$ & 1.30 & 5.38 & 10.4 & 16.17 & 24.37 \\
\hline $\mathrm{T} 6 \mathrm{~Hz}$ & 1.31 & 5.32 & 10.5 & 16.09 & 24.39 \\
\hline
\end{tabular}

Tabla 5 Media por banda de frecuencia en el grupo de PROMEDIO MENOR

\begin{tabular}{|l|r|r|r|r|r|}
\hline \multicolumn{1}{r}{ DELT } & \multicolumn{1}{c}{ THET } & \multicolumn{1}{c|}{ ALF } & \multicolumn{1}{c|}{ BETA } & \multicolumn{1}{c|}{ BETA } \\
\hline FP1Hz & 1.08 & 4.89 & 10.1 & 16.24 & 24.48 \\
\hline FP2Hz & 1.06 & 4.92 & 10.1 & 16.22 & 24.36 \\
\hline F3Hz & 1.19 & 5.11 & 10.0 & 16.19 & 23.98 \\
\hline F4Hz & 1.21 & 5.18 & 10.0 & 16.20 & 23.79 \\
\hline $\mathrm{C} 3 \mathrm{~Hz}$ & 1.22 & 5.10 & 10.1 & 16.25 & 24.03 \\
\hline $\mathrm{C} 4 \mathrm{~Hz}$ & 1.22 & 5.19 & 10.1 & 16.19 & 23.86 \\
\hline $\mathrm{P} 3 \mathrm{~Hz}$ & 1.22 & 5.14 & 10.3 & 16.04 & 23.81 \\
\hline $\mathrm{P} 4 \mathrm{~Hz}$ & 1.23 & 5.14 & 10.3 & 16.04 & 23.64 \\
\hline $\mathrm{O} 1 \mathrm{~Hz}$ & 1.22 & 5.06 & 10.5 & 15.83 & 23.70 \\
\hline $\mathrm{O} 2 \mathrm{~Hz}$ & 1.22 & 5.04 & 10.5 & 15.79 & 23.46 \\
\hline $\mathrm{F} 7 \mathrm{~Hz}$ & 1.1 & 5.01 & 10.1 & 16.31 & 24.41 \\
\hline $\mathrm{F} 8 \mathrm{~Hz}$ & 1.10 & 5.09 & 10.1 & 16.27 & 24.34 \\
\hline $\mathrm{T} 3 \mathrm{~Hz}$ & $1-17$ & 5.01 & 10.2 & 16.38 & 24.51 \\
\hline $\mathrm{T} 4 \mathrm{~Hz}$ & 1.18 & 5.10 & 10.2 & 16.32 & 24.41 \\
\hline $\mathrm{T} 5 \mathrm{~Hz}$ & 1.21 & 5.03 & 10.3 & 16.10 & 24.16 \\
\hline $\mathrm{T} 6 \mathrm{~Hz}$ & 1.21 & 5.06 & 0.42 & 16.06 & 23.96 \\
\hline
\end{tabular}

Tabla 6 Media por banda de frecuencia en el grupo de PROMEDIO MODERADO

\begin{tabular}{|l|l|l|l|l|l|}
\hline \multicolumn{2}{c}{ DELT } & \multicolumn{1}{c}{ THET } & \multicolumn{1}{c|}{ ALF } & \multicolumn{1}{c|}{ BETA } & BETA \\
\hline FP1Hz & 1.11 & 4.78 & 10.0 & 16.12 & 24.90 \\
\hline FP2Hz & 1.11 & 4.80 & 10.0 & 16.16 & 24.94 \\
\hline F3Hz & 1.18 & 5.17 & 9.96 & 16.18 & 24.22 \\
\hline F4Hz & 1.17 & 5.18 & 9.98 & 16.19 & 24.06 \\
\hline $\mathrm{C} 3 \mathrm{~Hz}$ & 1.18 & 5.23 & 10.0 & 16.21 & 24.12 \\
\hline $\mathrm{C} 4 \mathrm{~Hz}$ & 1.19 & 5.21 & 9.99 & 16.17 & 24.17 \\
\hline $\mathrm{P} 3 \mathrm{~Hz}$ & 1.18 & 5.12 & 10.2 & 16.09 & 23.98 \\
\hline $\mathrm{P} 4 \mathrm{~Hz}$ & 1.17 & 5.14 & 10.2 & 16.02 & 23.89 \\
\hline $\mathrm{O} 1 \mathrm{~Hz}$ & 1.18 & 5.03 & 10.6 & 15.79 & 23.96 \\
\hline $\mathrm{O} 2 \mathrm{~Hz}$ & 1.19 & 5.05 & 10.6 & 15.71 & 23.67 \\
\hline $\mathrm{F} 7 \mathrm{~Hz}$ & 1.14 & 4.97 & 10.1 & 16.30 & 24.80 \\
\hline $\mathrm{F} 8 \mathrm{~Hz}$ & 1.14 & 4.94 & 10.1 & 16.26 & 24.65 \\
\hline $\mathrm{T} 3 \mathrm{~Hz}$ & 1.18 & 5.06 & 10.1 & 16.40 & 24.85 \\
\hline $\mathrm{T} 4 \mathrm{~Hz}$ & 1.15 & 5.01 & 10.1 & 16.35 & 24.95 \\
\hline $\mathrm{T} 5 \mathrm{~Hz}$ & 1.19 & 5.00 & 10.3 & 16.03 & 24.28 \\
\hline $\mathrm{T} 6 \mathrm{~Hz}$ & 1.19 & 5.01 & 10.3 & 16.12 & 24.34 \\
\hline
\end{tabular}

Tabla 7 Media por banda de frecuencia en el grupo de PROMEDIO ALTO

ISSN-2523-2460

ECORFAN ${ }^{\circledR}$ Todos los derechos reservados

\section{Agradecimiento}

Se agradece a la Facultad de Medicina y Nutrición, así como al Instituto de Investigación Científica de la Universidad Juárez del estado de Durango por las facilidades otorgadas para la realización de la presente investigación.

\section{Conclusiones}

De las diferentes bandas de frecuencia electroencefalográfica, la actividad propia de la banda Alfa está definido que se encuentra entre los 8 y los $13 \mathrm{~Hz}$, lo cual regularmente se registra en sujetos sanos, despiertos, en reposo y con ojos cerrados, localizándose en la zona occipital y la amplitud normal de esta banda de frecuencia va de 20 a $200 \mu \mathrm{V}$ (Barea Navarro, 2014). Por lo anteriormente expresado se concluye que en el grupo estudiado, tanto por Estatus Escolar, como por Desempeño Escolar, la media de la frecuencia y de la amplitud de la actividad cerebral se observó dentro de parámetros de normalidad, es decir que funcionalmente desde la perspectiva electrofisiológica el cerebro de todos los participantes evaluados es normal.

La Correlaciona de Spearman con $\mathrm{p}<$ 0.05 confirma la hipótesis alterna identificándose que existe relación entre la actividad cerebral del rango THETA (de las áreas FP1, FP2, O1, F7, T3 y T4) y el promedio escolar, definiéndose que a mayor frecuencia Theta en las áreas referidas el promedio escolar es menor ó que a menor frecuencia Theta en las áreas referidas el promedio escolar es mayor.

El resultado de la correlación estadísticamente significativa brinda la oportunidad de plantear nuevos proyectos de investigación para identificar la forma en la que se pueda modificar la frecuencia Theta de la actividad cerebral en busca de disminuir su frecuencia esperando que esto se refleje en mejores promedios escolares. Queda entonces la tarea de buscar estrategias de intervención neurofisiológica y educativa que mejoren el desempeño escolar en los estudiantes universitarios. 


\section{Referencias}

Baker, R., \& al., e. (2010). Better to be frustrated than bored: The incidence, persistence ,and impact of learners cognitiveaffective states during interactions with three different computer-basedlearning environments. International Journal of Human-Computer Studies, 223-241.

Barceló, E. L. (2006). Funciones ejecutivas en estudiantes universitarios que presentan bajo y alto rendimiento académico. Piscología desde el Caribe , 109-138.

Barea Navarro, R. (16 de Marzo de 2014). Bioingenieria. Obtenido de Instrumentacion Biomédica:

http://www.bioingenieria.edu.ar/academica/cate dras/bioingenieria2/archivos/apuntes/tema\%205 $\% 20-\% 20$ electroencefalografia.pdf

Berka, C. e. (2007). EEG correlates of task engagement and mental workload in vigilance, learning and memory tasks. Aviation, Space, and Environmental Medicine , 231-244.

Casas Fernández C, B. A. (2002). Afectación cognitiva transitoria por actividad electroencefalográfica paroxística subclínica. Rev Neurol , 21-29.

Cupani, M. G. (2013). El modelo de los cinco grandes factores de personalidad: contribución predictiva al desempeño académico. Revista de Psicología, 17.

Damas Lopez, J., Martín Rodríguez, J., \& León Carrión, J. (2005). Patrón neurofisiológico del retraso mental: Estudio de un caso con electroencefalografía cuantitativa. Revista Española de Neuropsicología, 135-149.

Díaz Martínez C, D. M. (2006). Valor Del electroencefalograma en neonatología. Rev Mex Neuroci, 338-339.

Echeverria, M. G. (2007). Diferencias de género en habilidades cognitivas y rendimiento academico en estudiantes universitarios. Universidad Empresarial siglo 21.

Edel, R. (2003). El rendimiento académico: Concepto, investigación y desarrollo. Revista Electrónica Iberoamericana sobre calidad, eficacia y cambio en educación, s/p.
Flores Lázaro, J., \& Ostrosky Solis, F. (2008). Neuropsicología de Lóbulos Frontales, Funciones Ejecutivas y Conducta Humana. Revista Neuropsicología, Neuropsiquiatría y Neurociencias , 47-58.

Fuster, J. M. (2002). Frontal lobe and cognitive development. Neuropsychiatric Institute and Brain Research Institute, UCLA School of Medicine Los Angeles, California, USA, 37385 .

Gobierno de los Estado Unidos Mexicanos. (2017). 5o. Informe de Gobierno 2016-2017 (1 ed.). (P. d. REpública, Ed.) Ciudad de México: Talleres Gráficos de México.

Gobierno de los Estados Unidos Mexicanos. (2018). 6o. Informe de Gobierno. Ciudad de México: Talleres Gráficos de México.

Gobierno Federal de los Estados Unidos Mexicanos. (2015). \#er. Informe de Gobierno 2014-2015. Informe Anual, Gobierno Federal de los Estados Unidos Mexicanos, Presidencia de la República, Cd. de México.

Janzen, T., Graap, K., Stephanson, S., Marshall, W., \& Fitzsimmons, G. (1995). Differences in baseline EEG measures for ADD and Normally Achieving preadolescent males. Alberta, Canada: Kluwer Academic Publishers-Plenum Publishers.

Lubar, J. F., Rasey, H. W., McIntyre, A., Zoffuto, C., \& Abbott, P. L. (21 de Marzo de 2014). Nashville brain core. Obtenido de http://nashvillebraincore.info/research/Neurofee dback/eeg_training_college_students.pdf

Lutsyuk, N., É. E., \& Pavlenko, V. B. (2006). Correlation of the characteristics of EEG potentials with the indices of attention in 12- to 13- year old children. Neurophysiology , 209216.

Marosii, E., \& al., e. (2002). Narrow-band spectral measurements of EEG during emotional tasks. International Journal of Neuroscience, 871-891.

Mulas, F., Hernandez, S., Mattos, L., \& Abad Mas, L. E. (2006). Dificultades del aprendizaje en los niños epilépticos. Rev Neurol, 157-162.

Muñoz Céspedes, J. M., \& Tirapu Ustárroz, J. (2001). Rehabilitación Neuropsicológica. Madrid: Síntesis .

RIOS-VALLES, José Alejandro, BARRAGAN-LEDESMA, Laura Ernestina, ESTRADA-MARTÍNEZ, Sergio y POBLANO, Adrián. Relación entre el promedio escolar y las diferentes bandas de frecuencia del electroencefalograma de estudiantes de Medicina de la UJED campus Durango. Revista de Educación Técnica. 2019 
Niedermeyer, E., Lopes da Silva, F. H., \& Schomer, D. L. (2005). Electroencephalography: Basic principles, clinical applications, and related fields. USA: Lippincott Williams \& Wilki.

Palacios, J. \&. (2007). Desempeño académico y conductas de riesgo en adolescentes. Revista de Educación y Desarrollo, 5-16.

Portellano, J. A. (2005). Cómo desarrollar la inteligencia: entrenamiento neuropsicologico de la atencion y las funciones ejecutivas. España: McGraw Hill.

Rios Valles, J. A., Barragan Ledesma, L. E., \& Flores Saucedo, M. P. (14 de Noviembre de 2013). UJED. Obtenido de http://ujed.mx/Todo/documentos/pdf/eje_2/2_0 3.pdf

Rodriguez Ayán, M. N. (2011). Indicadores de rendimiento de estudiantes universitarios: calificaciones versus créditos acumulados. Revista de Educación , 467-492.

Salvatierra Belén, C. (2002). Aportaciones del electroencefalograma al diagnóstico $y$ tratamiento neuropsicológico de las dificultades del aprendizaje. Madrid: Universidad Complutense.

Secretaria de Educacion Publica. (2014). Recuperado el 08 de Septiembre de 2017, de http://www.planeacion.sep.gob.mx/:

http://www.planeacion.sep.gob.mx/Doc/estadist ica_e_indicadores/lineamientos_formulacion_d e_indicadores.pdf

Secretaria de Educación Pública, México Gobierno de la República. (2016). IV Informe de Labores 2015-2016. Secretaria de Educación Pública, Secretaria de Educación Pública. México: Impresora y Encuadernadora Progreso, SA de CV.

Universidad Juárez del Estado de Durango. (2015). Recuperado el 08 de 09 de 2017, de https://www.ujed.mx/:

https://www.ujed.mx/publicaciones/anuariosestadisticos
Universidad Juárez del Estado de Durango. (2016). Recuperado el 08 de 09 de 2017, de http://planeacion.ujed.mx/:

http://planeacion.ujed.mx/Documentos/Estadisti ca/Anuarios/anuario-estadistico-2016.pdf

Universidad Juárez del Estado de Durango. (2017). Anuario Estadistico 2017. Durango: UJED.

Universidad Juárez del EsTado de Durango. (2018). Anuario Estadístico 2018. Durango: UJED.

Velez-Van, M. R. (2005). Factors associated with academic performance in medical students. PSIC Educación Médica , 2 (8).

Ysunza, A., \& Perusquia Ortega, E. (2007). Electrodiagnóstico. Obtenido de http://www.medigraphic.com/pdfs/actmed/am2007/am072c.pdf 